\subsection{ERTAPENEM IS NON-INFERIOR TO CEFTRIAXONE FOR THE TREATMENT OF ANOGENITAL GONORRHEA: THE NABOGO RANDOMIZED DOUBLE BLIND NON- INFERIORITY TRIAL}

${ }^{1} \mathrm{H}$ de Vries ${ }^{*},{ }^{1} \mathrm{H}$ de Vries, ${ }^{1} \mathrm{~V}$ Jongen, ${ }^{1} \mathrm{~T}$ Heyman, ${ }^{2} \mathrm{C}$ Wind, ${ }^{1} \mathrm{~J}$ de Korne-Elenbaas, ${ }^{1} \mathrm{~A}$ van Dam, 'M Schim van der Loeff. 'Public Health Service (GGD) Amsterdam, Amsterdam, the Netherlands: ${ }^{2}$ Leiden UMC, Leiden, the Netherlands

10.1136/sextrans-2021-sti.165

Background Neisseria gonorrhoeae $(\mathrm{Ng})$ is a common sexually transmitted infection (STI). Emerging strains resistant to first-line ceftriaxone threaten $\mathrm{Ng}$ management. Hence, alternative treatments are needed. We evaluated the efficacy of ertapenem, gentamicin and fosfomycin as alternatives for $\mathrm{Ng}$.

Approach We included adults 18 years or older, with anorectal or urogenital gonorrhea in a randomized controlled, doubleblind, non-inferiority trial (three experimental- and one control-arm). Participants were randomized (1:1:1:1) to receive: intramuscular (IM) 500mg ceftriaxone, IM 1000mg ertapenem, IM $5 \mathrm{mg} / \mathrm{kg}$ gentamicin (maximum $400 \mathrm{mg}$ ), or $6 \mathrm{~g}$ fosfomycin orally. The primary outcome was the proportion of participants with a negative nucleic acid amplification test of the primary infected site, 7-14 days after treatment. Non-inferiority was established if the lower Hochberg-corrected 95\% confidence interval for difference between experimental and control arms was greater than $-10 \%$.

Outcomes Between 18 September 2017 and 5 June 2020, we assigned 346 participants to ceftriaxone $(n=103)$, ertapenem $(n=103)$, gentamicin $(n=102)$, and fosfomycin $(n=38)$. The fosfomycin arm was terminated early after interim analysis revealed $<60 \%$ efficacy. In the primary modified intent-totreat (mITT) analysis, all patients (93/93) in the ceftriaxone, $99 \%(86 / 87)$ in the ertapenem, 93\% (79/85) in the gentamicin, and $12 \%(4 / 33)$ in the fosfomycin arm cleared $\mathrm{Ng}$ [risk difference, ertapenem versus ceftriaxone, -0.01(95 CI: $0.06,0.03)$; gentamicin versus ceftriaxone -0.07 (95\%CI: -0.16 ,$0.005)]$. Both the secondary mITT analysis (clearance within 7-28 days), and the per-protocol analyses were consistent with the primary mITT analysis.

Significance Single-dose $1000 \mathrm{mg}$ ertapenem is non-inferior to single-dose $500 \mathrm{mg}$ ceftriaxone in gonorrhea treatment. Given that ertapenem, an already registered antibiotic, is non-inferior to the standard of care, it may currently provide an alternative treatment option for gonorrhea if resistance against ceftriaxone becomes more widespread.

\section{O20.3 QUANTITATIVE CHLAMYDIA TRACHOMATIS PGP3 SEROPOSITIVITY AND REPRODUCTIVE SEQUELAE AMONG WOMEN, NATIONAL HEALTH AND NUTRITION EXAMINATION SURVEY, UNITED STATES, 2013-2016}

${ }^{1} \mathrm{G}$ Anyalechi*, 1J Hong, 'D Danavall, ${ }^{2} \mathrm{D}$ Martin, ${ }^{2} \mathrm{~S}$ Gwyn, ${ }^{3} \mathrm{P}$ Horner, ${ }^{1} \mathrm{~B}$ Raphael, ${ }^{1} \mathrm{R}$ Kirkcaldy, ${ }^{1} \mathrm{E}$ Kersh, ${ }^{1} \mathrm{~K}$ Bernstein. ${ }^{1}$ Division of STD Prevention, Centers For Disease Control and Prevention, Atlanta, USA; ${ }^{2}$ Division of Parasitic Diseases, Centers for Disease Control and Prevention, Atlanta, USA; ${ }^{3}$ Population Health Sciences and National Institute for Health Research, Health Promotion Research Unit in Behavioural Science and Evaluation in Partnership with Public Health England, University of Bristol, Bristol, UK

10.1136/sextrans-2021-sti.166

Background Chlamydia trachomatis infection causes reproductive sequelae of pelvic inflammatory disease (PID) and tubal infertility. Previous studies have demonstrated a relationship between levels of chlamydia antibody and these sequelae. We investigated associations of high chlamydial seropositivity with PID and infertility using a Pgp3 antibody (Pgp3Ab) multiplex bead array (Pgp3AbMBA).

Methods We performed chlamydia Pgp3AbMBA on sera from women 18-39 years old participating in the 2013-2016 National Health and Nutrition Examination Survey with urine chlamydia nucleic acid amplification test results. High chlamydial seropositivity was defined as a median fluorescence intensity $(\mathrm{MFI}) \geq 50,000$; low-positive was $\mathrm{MFI}>551-<50,000$; negative was $<551$. Weighted US population high-positive, low-positive, and negative Pgp3Ab chlamydia seroprevalence and 95\% confidence intervals (CI) were calculated for women with chlamydia, PID and infertility.

Results Sera were available from 1,725 (73.7\%) of 2,339 women aged 18-39 years; 1,425 were sexually experienced. Overall, 104 women had high-positive Pgp3Ab (5.4\% [95\% CI $4.0-7.0]$ of US women); 407 had low-positive Pgp3Ab (25.1\% [95\% CI 21.5-29.0]), and 914 were negative $(69.5 \%$ [95\% CI 65.5-73.4]). The prevalence of current chlamydia among women with high seropositivity (10.7\% [95\% CI 5.418.6]) was higher than the prevalence of current chlamydia among seronegative women (0.4\% [95\% CI 0.1-1.3]).

Among women with high-positive Pgp3Ab, infertility prevalence was 2.0 (95\% CI 1.1-3.7) times that among Pgp3Ab-negative women (19.6\% [95\% CI 10.5-31.7] versus 9.9\% [95\% CI 7.7-12.4]). For women with low-positive Pgp3Ab, PID prevalence was 7.9\% (95\% CI 4.6-12.6) compared to $2.3 \%(95 \%$ CI $1.4-3.6)$ in those with negative Pgp3Ab.

Conclusions High chlamydial Pgp3 Ab seropositivity was associated with infertility. Small sample size limited evaluation of an association of high seropositivity with PID. In infertile women, Pgp3 Ab may be a marker of prior chlamydia.

\section{O20.4 EVALUATION OF GLOBAL TREPONEMA PALLIDUM GENETIC DIVERSITY USING REPRESENTATIVE SAMPLING AND HIGH-THROUGHPUT GENOMIC ANALYSIS}

${ }^{1} \mathrm{~F}$ Nindo*, ${ }^{2} \mathrm{P}$ Pospisílová, ${ }^{1} \mathrm{C}$ Hennelly, ${ }^{3,4} \mathrm{~K}$ Hawley, ${ }^{4} \mathrm{~A}$ Luthra, ${ }^{3} \mathrm{~T}$ Davenport, ${ }^{1} \mathrm{~A}$ Sena ${ }^{5} \mathrm{~W}$ Chen, ${ }^{5} \mathrm{Y}$ Jiang, ${ }^{5} \mathrm{~B}$ Yang, ${ }^{5} \mathrm{~L}$ Yang, ${ }^{5} \mathrm{H}$ Zheng, ${ }^{6} \mathrm{M}$ Matoga, ${ }^{1,6} \mathrm{I}$ Hoffman, ${ }^{7} \mathrm{E}$ Lopez, ${ }^{7,8} \mathrm{~L}$ Ramirez, ${ }^{3} \mathrm{M}$ Caimano, ${ }^{3,4} \mathrm{~J}$ Salazar, ${ }^{9} \mathrm{M}$ Moody, ${ }^{3,4} \mathrm{~J}$ Rudolf, ${ }^{1 \mathrm{~J}} \mathrm{Juliano},{ }^{2} \mathrm{D}$ Smajs, ${ }^{\mathrm{J} J}$ Parr. ${ }^{1}$ University of North Carolina (UNC) at Chapel Hill, Chapel Hill, USA; ${ }^{2}$ Masaryk University, Brno, Czech Republic; ${ }^{3}$ UConn Health, Farmington, USA; ${ }^{4}$ Connecticut Children's Hospital, Hartford, USA; ${ }^{5}$ Southern Medical University Dermatology Hospital, Guangzhou, China; ${ }^{6}$ UNC Project Malawi, Lilongwe, Malawi; ${ }^{7}$ CIDEIM, Cali, Colombia; ${ }^{8}$ Universidad Icesi, Cali, Colombia; ${ }^{9}$ Duke University, Durham, USA

\subsection{6/sextrans-2021-sti.167}

Background Large-scale genomic analyses of Treponema pallidum (Tp) are now possible using enrichment methods that enable sequencing without in vivo cultivation in rabbits. Using capture and amplification enrichment approaches, we are generating complete genomes from diverse clinical samples collected as part of a multi-site syphilis vaccine development project. Analysis of these and a growing number of publicly available genomes provides new insights into the global diversity and evolution of $\mathrm{Tp}$.

Methods Clinical samples were collected from subjects presenting with primary and secondary syphilis in the US, Columbia, Malawi, China, and Czech Republic. DNA extracted from 